\section{MS8-O2 Crystal structure of the influenza A polymerase bound to the viral RNA promoter}

Alexander Pflug ${ }^{1}$, Delphine Guilligay ${ }^{1}$, Stefan Reich ${ }^{1}$, Stephen Cusack $^{1}$

\section{EMBL Grenoble}

email: apflug@embl.fr

According to the World Health Organization the influenza virus causes 250,000 to 500,000 deaths per year during its seasonal outbreak. Moreover the virus bears the potential to cause devastating pandemics with several million casualties when it evolves rapidly through reassortment between different strains; as happened with the 'Spanish flu' in 1918. During an infection influenza relies on its own polymerase to replicate its genome and transcribe mRNAs, encoding for the viral proteins that pack and wrap the viral genome copies to form new infectious virus particles. The influenza polymerase is a $250 \mathrm{kDa}$ heterotrimeric protein-complex composed of the subunits PA, PB1 and PB2, with PB1 constituting the actual RNA-dependent RNA-polymerase (RdRp).

We present the crystal structure of the influenza $A$ polymerase bound to its viral RNA promoter [1]. The structure depicts a molecule with a canonical viral RdRp in its core (protein PB1), closely intertwined with the proteins PA and PB2 (Figure 1). The novel structural information on the PB1 protein opens the door for a new era of structure-based drug development of RdRp-targeted anti-influenza agents. Homologous polymerases from other pathogenic viruses are used as drug targets successfully, as for instance the reverse transcriptase from HIV and the RNA polymerase from hepatitis $\mathrm{C}$ virus. It became now possible to apply this approach in case of influenza; not only because this is the first report of a structure of the influenza PB1 protein, but also because this is the first report of the recombinant production of enzymatically active PB1 protein in sufficient amounts for in vitro drug screenings. The structure of the polymerase complex gives important insight into the mechanism of this elaborate molecular machine. It depicts the binding to the RNA promoter, the conserved ends of the single-stranded genomic RNA segments, and it depicts how the PA endonuclease domain and PB2 cap-binding domain interact to achieve the so called cap-snatching of the host cell mRNA.

[1] Pflug A, Guilligay D, Reich S, Cusack S. (2014) Nature, 516, 355-360

\section{proteins PA, PB1 (RdRp), PB2}

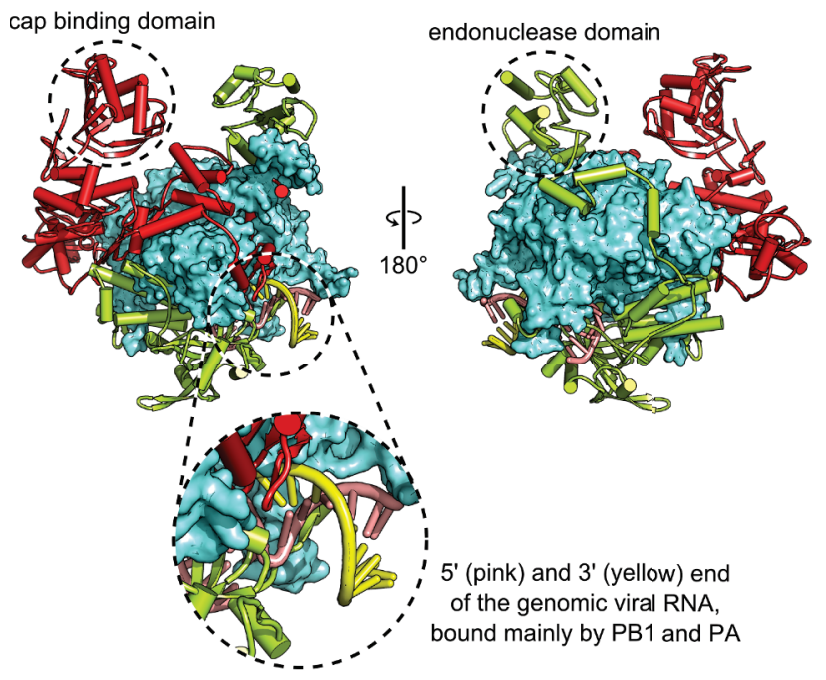

Figure 1. Overall architecture of the influenza polymerase complex. The RNA-dependent RNA-polymerase (RdRp) forms the core of the molecule, wrapped by the proteins $\mathrm{PA}$ and $\mathrm{PB} 2$ (PDB ID: 4WSB).

Keywords: influenza, polymerase, RNA, virus 